Norris, K. P. \& Greenstreet, J. E. S. (1958). J. gen. Microbiol. 19, 566-580

\title{
On the Infrared Absorption Spectrum of Bacillus megaterium
}

\author{
By K. P. NORRIS aNd J. E. S. GREENSTREET
}

Microbiological Research Establishment, Ministry of Supply, Porton, Wiltshire

SUMMARY: The infrared absorption spectra of the aerobic spore-forming bacilli are variable. Evidence is presented to show that, for Bacillus megaterium, the nature of the spectrum is related to the morphology of the bacteria, both showing a cyclic variation with time. The time scale of the cycle is affected by the temperature at which the cultures are incubated, by their population density and by the medium on which they are grown; it may be affected by induced drug resistance. Some of the characteristics of the spectrum of vacuolated vegetative organisms are due to the presence of an extractable substance which may be a polymer of $\beta$-hydroxybutyric acid. Difference spectroscopy is used to demonstrate the presence of this substance, its disappearance as the bacteria autolyse and the appearance of $\beta$-hydroxybutyric acid in the autolysed material. This technique is applied also to establish the state of dipicolinic acid in the resting spores of $B$. megaterium. Any application of infrared spectroscopy to the study of aerobic spore-forming bacilli must take account of the morphological state of the bacteria.

It has been demonstrated that, for strains representative of many genera, reproducible infrared absorption spectra can be obtained from films of bacteria if the conditions of growth, the methods of harvesting and specimen preparation, and the instrument conditions are carefully standardized (Thomas \& Greenstreet, 1954; Riddle et al. 1956). The spectra of the aerobic spore-forming bacilli are not nearly so reproducible (Blackwood \& Epp, 1957; Haynes, Melvin, Locke \& Glass, 1958) and in some instances have more absorption bands than those of other genera. In our previous investigation (Greenstreet \& Norris, 1957) it was found that the spectrum of Bacillus megaterium underwent a regular change preceding the formation of spores. The changes in spectrum of one species of Bacillus are much greater than the reproducible differences observed between other strains, species or genera. This fact makes it difficult to fit the aerobic spore-forming bacilli into a system of classification based upon their infrared absorption spectra.

It is the object of this paper to study the changes in spectrum of Bacillus megaterium and to show that they are connected with the morphological appearance of the organisms and with the presence in the organisms of substances which have been isolated and characterized. The relevance of these changes in spectra to the differentiation of the aerobic spore-forming bacilli is discussed.

\section{METHODS}

Those workers principally interested in differentiating bacteria (Randall. Smith, Colm \& Nungester, 1951; Stevenson \& Bolduan, 1952; Thomas \& Greenstreet, 1954; Levine, Stevenson, Bordner \& Edwards, 1955; Riddle et al. 1956; Greenstreet \& Norris, 1957; Kenner, Riddle, Rockwood \& Bord- 
ner, 1958) have insisted upon the use of standardized procedures; we have tried to do the same. For most of the experiments Hills's glucose lactate agar medium (GL agar) was used (Hills, Belton \& Blatchley, 1949). Tryptic meat agar (TM agar), tryptose agar and liquid potato casein yeast medium (potato CCY; Powell \& Strange, 1956) were also used.

A laboratory strain of Bacillus megaterium was used for most of the experiments. A limited investigation was made of laboratory strains of $\boldsymbol{B}$. anthracis, B. cereus, B. pumilus and B. subtilis. Seed was stored at $3^{\circ}$ as a resting spore suspension and watch was kept for contamination. For an experiment requiring 100 plates $(9 \mathrm{~cm}$. diameter Petri dishes), the procedure for preparing samples of bacteria for infrared examination was broadly as follows. The inoculum for the plates was prepared by adding $0.75 \mathrm{ml}$. spore suspension $\left(2 \cdot 3 \times 10^{10}\right.$ spores $/ \mathrm{ml}$.) to $100 \mathrm{ml}$. liquid medium (tryptic meat broth) contained in each of four Erlenmeyer flasks of capacity $500 \mathrm{ml}$. These were shaken at $37^{\circ}$ for $70 \mathrm{~min}$. so as to produce a suspension of germinated spores or early vegetative organisms with a uniform population. That is to say, all the organisms were similar in appearance. The shaking machine operated at $90 \mathrm{cyc} . / \mathrm{min}$. and had a stroke of $3 \mathrm{in}$. The suspensions were then centrifuged at $1900 \mathrm{~g}$ for $30 \mathrm{~min}$. in a refrigerated centrifuge, and the deposit was resuspended in a volume of distilled water or physiological saline such that a predetermined density was obtained. Each plate of the selected solid medium was inoculated with $0.5 \mathrm{ml}$. of this suspension. A viable count (Miles \& Misra, 1988) was made on the suspension used to inoculate the plates. The details of the procedure were varied to suit particular experiments, but the inoculum for the plates was always of germinated spores or early vegetative organisms with as uniform a population as possible. After incubation for various periods at the chosen temperature the bacteria were washed off the plates, centrifuged and resuspended in saline. Some of the suspensions were examined alive and some after autoclaving at $20 \mathrm{lb}$./sq.in. for $30 \mathrm{~min}$.

The morphology of the bacteria was assessed by microscopical examination of films. These were made from the suspension used to inoculate the plate cultures and from the various suspensions harvested from them immediately before killing. They were stained to demonstrate and distinguish spores (Powell, 1950) and to demonstrate fat globules (Hartman, 1940). Occasionally wet films were examined under phase contrast illumination, since in this way refractile granules are readily visible (Hewitt, 1951).

A fixed volume of the bacterial suspension was spread evenly on a silver chloride window of prescribed size and heated at $60^{\circ}$ until a dry film was obtained. This film was placed in the sample beam of the spectrophotometer and a similar but clean piece of silver chloride in the reference beam. Some suspensions were freeze dried. The spectra of these were obtained by preparing pressed disks containing about $1 \%$ of the freeze-dried material in A.R. potassium chloride (Ford \& Wilkinson, 1954).

The absorption spectra were recorded between 2 and $15 \mu$ as $\%$ transmission versus wavelength by using a Grubb-Parsons $\mathrm{S} 4$ double-beam spectrophotometer equipped with a rock-salt prism. 


\section{RESULTS}

We have examined the effect of varying the following factors upon the spectrum and morphology of Bacillus megaterium: (1) duration of incubation of the cultures; (2) temperature of incubation; (3) population density; (4) nature of the medium; (5) induced drug resistance.

\section{Variation of spectrum with duration of incubation of the cultures}

Plates of GL agar were each inoculated with $0.5 \mathrm{ml}$. of a suspension $\left(2 \times 10^{8}\right.$ viable early vegetative organisms $/ \mathrm{ml}$.) of Bacillus megaterium and incubated at $37^{\circ}$. At intervals suspensions were prepared by washing off the growth from different plates. Dried films of these suspensions gave spectra (Fig. 1) which show a regular change depending upon the duration of incubation. The change consists of absorption bands appearing in the spectrum at $5 \cdot 78,7 \cdot 68,7 \cdot 97,8 \cdot 46,8 \cdot 85,9 \cdot 11,9 \cdot 47,10 \cdot 22$ and $12 \cdot 10 \mu$. These bands increase in intensity, reach a maximum at about $5 \mathrm{hr}$. and then decrease as the dura-

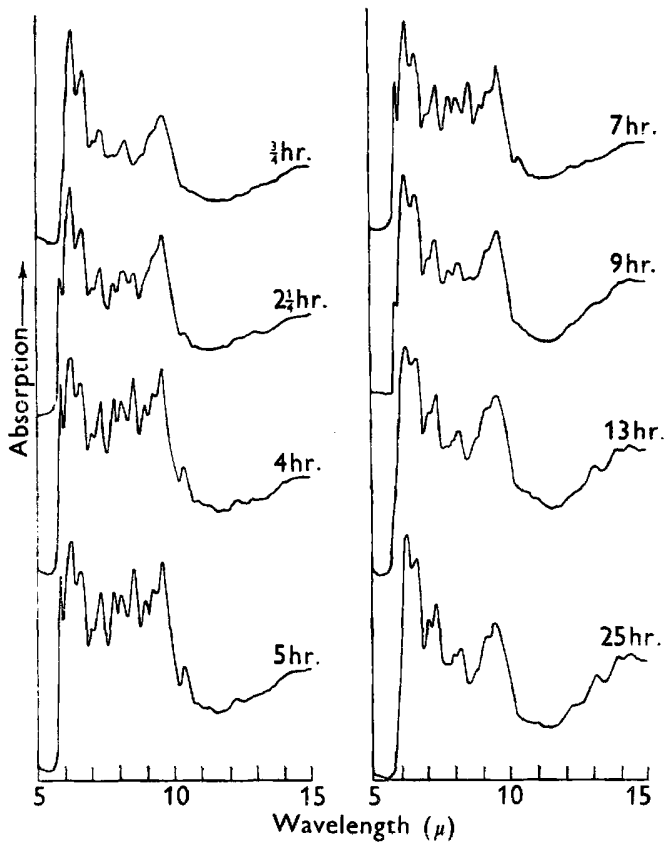

Fig. 1

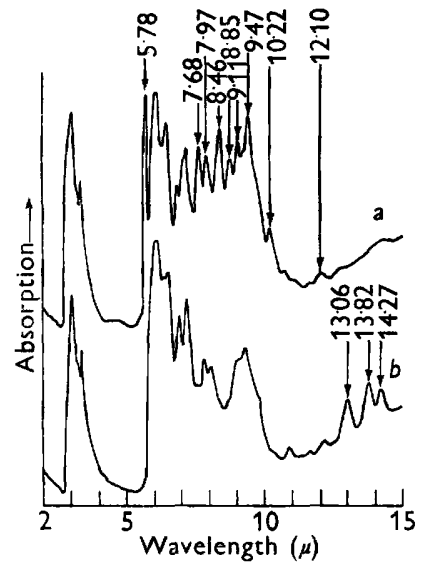

Fig. 2

Fig. 1. Variation of the infrared absorption spectrum of Bacillus megaterium with duration of incubation. Nine $\mathrm{cm}$. diameter plates of Hills's glucose lactate medium were each inoculated with $10^{8}$ early vegetative organisms and incubated at $37^{\circ}$. $\frac{3}{4} \mathrm{hr} .=$ early vegetative organisms. $2 \frac{1}{\mathrm{hr}}$.= vegetative organisms showing signs of vacuolation. 4-5 hr. = highly vacuolated vegetative organisms. $7 \mathrm{hr} .=$ prespores forming in cells. $9 \mathrm{hr},=$ prespores. $13-25 \mathrm{hr} .=$ resting spores.

Fig. 2. Infrared absorption spectra of Bacillus megaterium. a, vacuolated vegetative organisms; $b$, resting spores. The absorption bands indicated are those referred to in the text: their wavelengths are given in microns. 
tion of incubation increases. A spectrum with these additional absorption bands, which are indicated in Fig. $2 a$, will be described as complex. As these bands decrease in intensity new absorption bands appear at 13.06, 13.82 and 14.27 $\mu$ (Fig. $2 b$ ).

Films of the cultures stained with sudan black and with carbol fuchsinemethylene blue had the following appearances. After $\frac{3}{4} \mathrm{hr}$. incubation there was little detectable uptake of sudan black and with the spore stain the organisms appeared uniformly deep blue. After $2 \frac{1}{4} \mathrm{hr}$. each organism contained several small granules which stained with sudan black; with the spore stain each organism contained several bodies which took up less blue stain than the remainder of the bacterium. At this stage the spectrum was becoming complex. Between 4 and $5 \mathrm{hr}$. the average length of the organisms decreased and they took up more sudan black than before although the granules were not so well defined. With the spore stain the organisms appeared to be highly vacuolated, the vacuoles occupying a large part of the bacterium. Organisms at this stage of morphological development will subsequently be referred to as vacuolated vegetative organisms. The spectrum of such organisms was complex (Fig. 1). After $7 \mathrm{hr}$. incubation the sudan black-staining granules had become very diffuse, the material staining blue with the spore stain was tending to concentrate at the poles and each organism contained one large pink-staining body. By $9 \mathrm{hr}$. there was hardly any uptake of sudan black, the prespore was well developed and stained a delicate pink with the spore stain and the remnants of the organism appeared as blue-staining material at the poles of the prespore. The spectrum of such organisms was less complex than at $5 \mathrm{hr}$. After $13 \mathrm{hr}$. the culture was entirely of free spores or of well-developed prespores which took up no sudan black. The spectrum was no longer complex and the bands shown in Fig. $2 b$ were present. After $25 \mathrm{hr}$. incubation the culture consisted entirely of resting spores. This brief description agrees well with the more detailed observations of sporing cultures by Bayne-Jones \& Petrilli (1933) and Lewis (1934).

Repeated experiments confirmed that there is an association between the shape of the spectrum and the morphology of the bacteria. Early vegetative organisms which stain uniformly with methylene blue do not have complex spectra. Vacuolated vegetative organisms have complex spectra. The prespore and resting spore do not have complex spectra, the spectrum of resting spores having the extra absorption bands at 13.06, 13.82 and 14.27 $\mu$.

Cultures and spectra of Bacillus anthracis and B. cereus underwent a similar cyclic change. $B$. subtilis and $B$. pumilus also exhibited a cyclic change of spectrum with duration of incubation, but this differed from that for $B$. megaterium. At no stage was the spectrum complex, but the absorption bands at 13.06, 13.82 and $14 \cdot 27 \mu$ appeared in the spectrum of old cultures.

Changes in the spectra of Bacillus megaterium were characterized by the ratio of the strengths of two selected absorption bands. For this purpose strength is defined as the optical density at the band peak minus the optical density of the background at the same wavelength. The background is taken to be a straight line drawn tangential to the minima in the absorption spectra 
near 5.5 and $11.5 \mu$. The bands chosen were (1) a band at $6.10 \mu$ which appeared to remain constant throughout the cycle of events; (2) a band at $7 \cdot 68 \mu$ which varied markedly with the duration of incubation and attained a maximum at the same time as the other eight bands in the complex spectrum.

The ratio $\alpha$ is defined by

$$
\alpha=\frac{\text { strength of band at } 7 \cdot 68 \mu}{\text { strength of band at } 6 \cdot 10 \mu} \text {. }
$$

Plotting the ratio $\alpha$ against duration of incubation for the spectra shown in Fig. 1 gives a curve with a maximum at $5 \mathrm{hr}$. Whenever cultures of Bacillus megaterium gave spectra with a high $\alpha$ the organisms appeared vacuolated with the spore stain and contained granules which stained with sudan black.

In an attempt to relate the change of $\alpha$ with the rate of growth of Bacillus megaterium, spores were inoculated into shaken potato CCY and at intervals the culture was sampled. The dry weight and $\alpha$ for each sample were plotted against time. In the early stages of the logarithmic phase of growth, when the bacteria stained uniformly with methylene blue, $\alpha$ was small; in the later stages, when the organisms were both larger and increasingly vacuolated, $\alpha$ increased. The dry weight and $\alpha$ each reached their maximum at about the same time. After this $\alpha$ fell rapidly whereas the dry weight fell slowly.

\section{Variation of spectrum with temperature of incubation of culture}

A large number of plates of GL agar were each inoculated with $0.5 \mathrm{ml}$. of a suspension $\left(4 \times 10^{7}\right.$ viable early vegetative organisms $/ \mathrm{ml}$. $)$ of Bacillus megaterium. Half of these plates were incubated at $37^{\circ}$ and half at $20^{\circ}$. Cultures incubated at the two temperatures were harvested at intervals until spores were formed. The values of $\alpha$ obtained from the spectra, when plotted against duration of incubation, gave curves of similar shape for each temperature, but with the maxima occurring after different periods of incubation: cultures grown at $37^{\circ}$ had peak $\alpha$ at $5 \frac{1}{2} \mathrm{hr}$., whereas cultures grown at $20^{\circ}$ had peak $\alpha$ at $24 \mathrm{hr}$. (Fig. 3). Bacteria from the cultures which gave maximum $\alpha$ at the two temperatures were alike in appearance and could be described as vacuolated vegetative. The bacteria grown at the lower temperature remained longer at the stage of development at which they stained uniformly with methylene blue. Similar results were obtained when the experiment was repeated at $37^{\circ}$ and $\mathbf{2 3}^{\circ}$.

\section{Variation of spectrum with population density}

Plates ( $9 \mathrm{~cm}$. diameter) of GL agar were inoculated evenly with $10^{8}, 10^{7}$, $10^{6}, 10^{5}, 10^{4}$ and $10^{3}$ organisms/plate and incubated at $37^{\circ}$. After $10 \mathrm{hr}$. incubation confluent growth was obtained on the plates with inocula of $10^{5}$ or greater. Curves of $\alpha$ versus duration of incubation for cultures grown from the different inocula are shown in Fig. 4. Similar curves were obtained when tryptose agar was used instead of GL agar. As the inoculum decreased so the time required for $\alpha$ to reach a maximum increased. Organisms on the plates with the smaller inocula remained in the early vegetative stages of growth 
longer than those on plates with a larger inoculum. At peak $\alpha$ the bacteria had the same morphological appearance for all inocula. The effect that a change of inoculum can have upon the assessment of spectra is best demonstrated by an example. After $10 \frac{1}{2} \mathrm{hr}$. incubation, cultures grown from inocula of $10^{6}$ and $10^{5}$ organisms/plate had almost identical spectra which had the same value of $\alpha$. Three hours later spectra from the two cultures were very

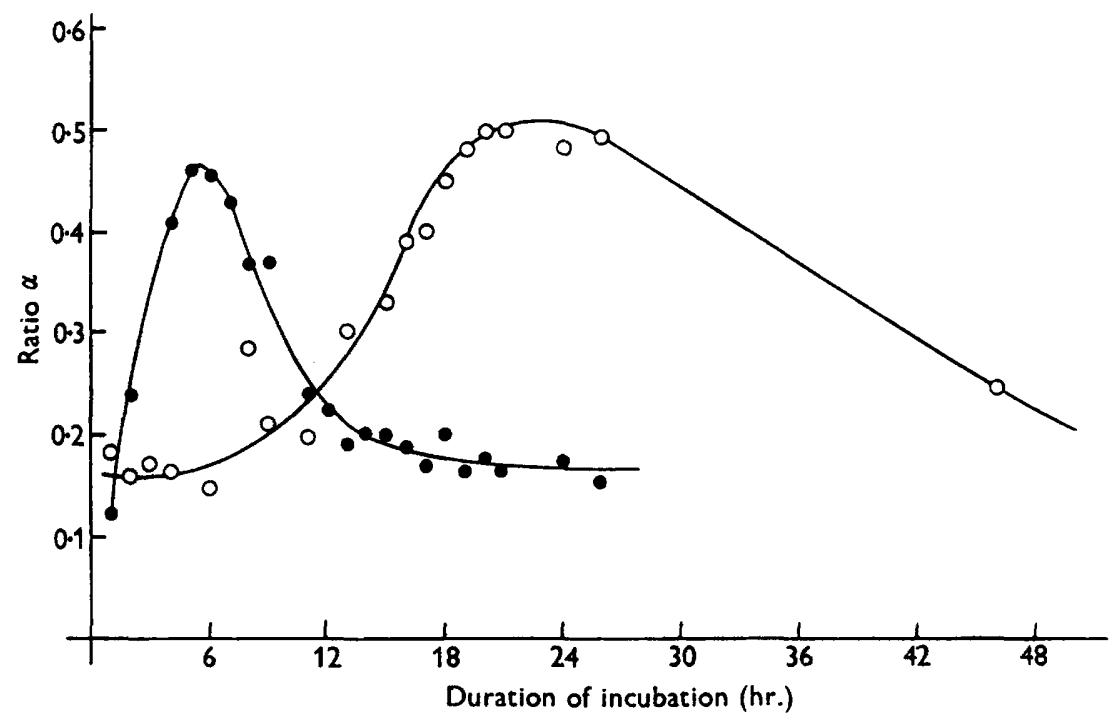

Fig. 3. Curves showing the change of $\alpha$ with duration of incubation for Bacillus megaterium grown on Hills's glucose lactate medium. $O-O$ at $20^{\circ} ;-$ at $37^{\circ}$; the inoculum was the same for all plates.

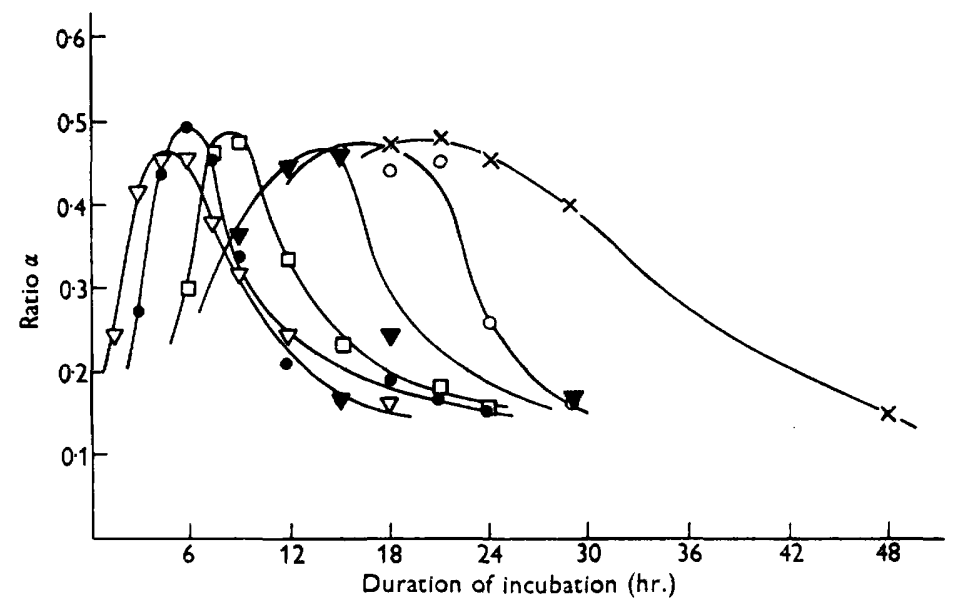

Fig. 4. Curves showing the change of $\alpha$ with duration of incubation for Bacillus megaterium grown on Hills's glucose lactate medium at $37^{\circ}$. The inoculum was varied: $\nabla-\nabla, 10^{8}$; $\longrightarrow, 10^{7} ; \square-\square, 10^{6} ; \nabla-\nabla, 10^{5} ; O^{-}-O, 10^{4} ; x-x, 10^{8}$ viable organisms/plate (9 cnl. diameter). 
different indeed. The spectrum of the culture resulting from the inoculation of $10^{5}$ organisms/plate had all the absorption bands shown in Fig. $2 a$ and $\alpha$ was at its peak, whereas the spectrum of the culture from the $10^{6}$ inoculum lacked these bands and gave a low value of $\alpha$. A tenfold change in inoculum thus had a great effect upon the rate of development of the culture.

\section{Variation of spectrum with medium}

We used GL agar for the above experiments because we found that Bacillus megaterium grew uniformly on this medium and spore formation proceeded at a uniform rate throughout the culture, i.e. microscopic examination at any time showed that the majority of the bacteria in the culture had reached the same stage of development. Dilution of vacuolated vegetative organisms with non-vacuolated organisms reduced the value of $\alpha$ and made it more difficult to follow the changes described above.

Growth of Bacillus megaterium in shaken liquid potato CCY medium gave similar results to growth on plates of GL agar although in general the peak $\alpha$ for the spectra of bacteria grown in shaken liquid cultures was greater than that for cultures on solid media.

Bacillus megaterium grown on TM agar did not proceed beyond the vegetative phase of growth. The organisms when stained with the spore stain appeared vacuolated at first and then the vacuoles decreased in size but no spores were produced. The spectra showed a series of changes similar to that for growth on GL agar, the ratio $\alpha$ reaching a maximum when the bacteria were most vacuolated. When $1 \%(w / v)$ glucose was added to the medium both the bacteria and the vacuoles appeared to be larger and the maximum value of $\alpha$ was reached after a longer period of incubation. The addition of $2 \%$ $(\mathrm{w} / \mathrm{v})$ glucose to TM agar increased the effect produced by adding $1 \%$, but again no spores were formed.

On tryptose agar the morphology developed in a similar way to that on GL agar. Spores were produced after the vacuolated vegetative stage. The addition of glucose delayed spore formation. The greater the amount of added glucose the longer it took for $\alpha$ to reach a maximum.

Cultures from TM agar, tryptose agar and GL agar with added glucose harvested after a period of incubation greater than $10 \mathrm{hr}$. (inoculum 10? viable organisms/plate, temperature $37^{\circ}$ ) had a more complex spectrum than those harvested from the medium without added glucose.

\section{Variation of spectrum with induced drug resistance}

The tolerance of the parent strain of Bacillus megaterium to various antibiotics was increased by repeated subcultivation on gradient plates containing the antibiotics (Szybalski \& Bryson, 1952). In this way tolerance to chloramphenicol, aureomycin, terramycin, streptomycin and bacitracin was increased. The relative tolerances of the parent strain and the derived strains to each antibiotic were assessed by a tube dilution method.

Cultures of the parent and of derived strains resistant to chloramphenicol, aureomycin, terramycin and streptomycin were harvested at intervals from 
plates of GL agar, which had been inoculated with the same number of viable organisms/plate $\left(5 \times 10^{6}\right)$ and incubated at the same temperature. The spectra of these five series of samples were almost identical for any one period of incubation.

Small differences between spectra at any one time could be explained by differences in the morphology of the bacteria. The parent strain and the strain resistant to bacitracin were compared in a similar experiment. The curve of $\alpha$ plotted against duration of incubation for the bacitracin-resistant derivative was very different from that for the parent, its maximum appearing much later. When harvested after periods greater than $10 \mathrm{hr}$. the bacitracin-resistant strain always showed a more complex spectrum than the parent. When, however, the spectra were compared on the basis of morphology the peak value of $\alpha$ always coincided with the same stage of morphological development, i.e. vacuolated vegetative organisms. The difference between the spectra reflects a difference between the growth rates of the parent and derived strain. The difference in the rate of development of the cultures was obvious by even a cursory examination of the plates.

\section{Chemical examination of vacuolated vegetative cells}

The evidence presented by Burdon, Stokes \& Kimbrough (1942) and Knaysi (1946) suggested that inclusions in vegetative organisms of some species of the genus Bacillus are fat-like. Attempts were made to extract such a substance from vacuolated vegetative organisms of $B$. megaterium by using acetone, benzene, carbon tetrachloride, chloroform, diethyl ether, ethanol and methanol. We did not succeed in extracting any fat or the component responsible for the extra absorption bands. In the course of making cell-wall preparations of vacuolated vegetative organisms by differential centrifugation of bacteria disintegrated by shaking with ballotini, a fraction was isolated which had an infrared absorption spectrum with peaks at all the wavelengths marked in Fig. $2 a$. The spectrum of the cell walls was almost entirely free from the bands due to this fraction, showing that it is contained within the bacterium and is not part of the wall.

Lemoigne (1923) found that suspensions of vegetative organisms in the Bacillus subtilis group became increasingly acid during autolysis owing to the formation of $\beta$-hydroxybutyric acid. A study of this phenomenon led him to isolate from $B$. megaterium and from $B$. mesentericus a substance which he referred to as 'lipide $\mathrm{Y}$ ' and which he concluded was a polymer of $\beta$-hydroxybutyric acid (Lemoigne, 1926). He eventually succeeded in isolating two different products with melting points of $154^{\circ}$ and $120^{\circ}$ and he suggested that they were both polymers of $\beta$-hydroxybutyric acid but with different degrees of polymerization.

We have used one of his methods to isolate from vacuolated vegetative organisms of Bacillus megaterium a substance which corresponds with his 'lipide Y'. For convenience we shall refer to the material we isolated as the 'lipid'. The mean yield was $16 \%$ of the dry weight of the bacteria when these were grown on GL agar and $19 \%$ in potato CCY.

The physical and chemical properties of the lipid isolated from our strain 
were similar to those described by Lemoigne for the 'lipide' he isolated; our lipid contained $<0.02 \%$ phosphorus. The only exception was the melting point of the lipid. Our product melted at $165^{\circ}$, whereas Lemoigne's products melted at $154^{\circ}$ and $120^{\circ}$. Lemoigne said that the 'lipide' is a polymer of $\beta$-hydroxybutyric acid and the infrared spectrum is not at variance with this suggestion. Perhaps the product isolated by us had a higher degree of polymerization than either of those isolated by Lemoigne.

\section{Difference spectroscopy applied to Bacillus megaterium}

Spectra of vacuolated organisms and germinated spores have much in common (Fig. 1). By placing a specimen of vacuolated organisms in the sample beam of a double-beam instrument and germinated spores in the reference beam it is possible to obtain the spectrum of the difference between the two

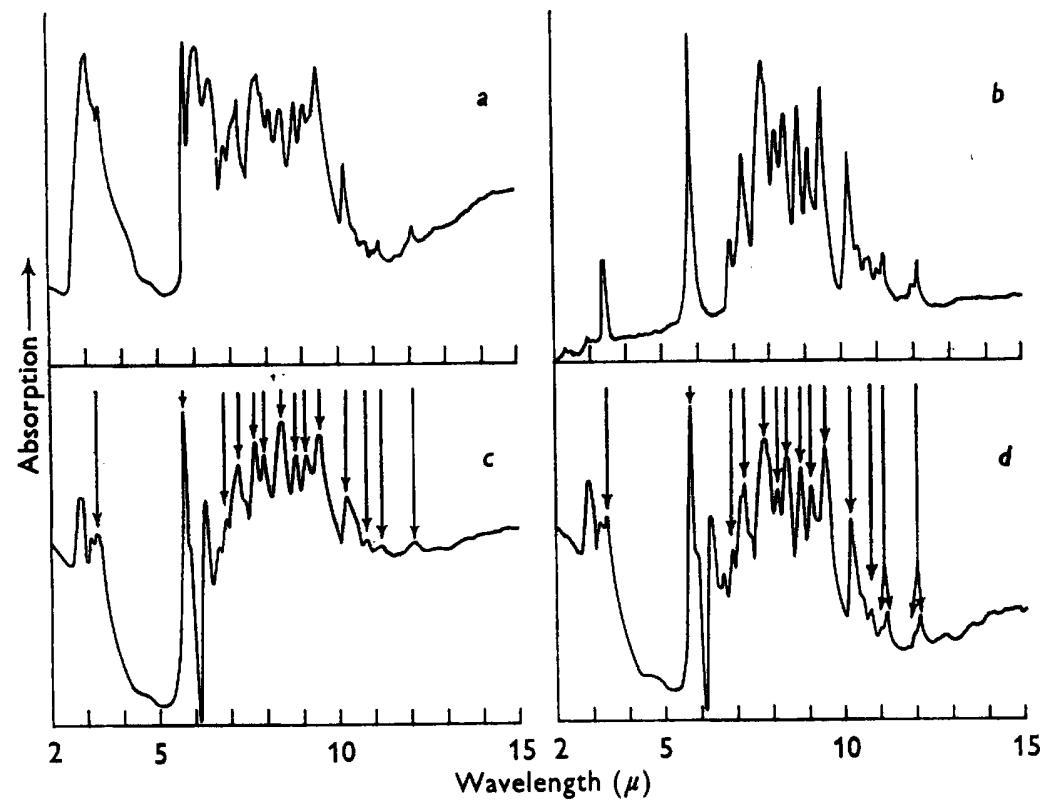

Fig. 5. Infrared absorption spectra of Bacillus megaterium. a, type $\mathbf{B}$ spectrum of vacuolated vegetative organisms; $b$, the lipid isolated from vacuolated vegetative organisms (dried film on silver chloride); $c$, the difference spectrum between vacuolated vegetative organisms which have a type $\mathbf{A}$ spectrum, and germinated spores; $d$, the difference spectrum between vacuolated vegetative organisms which have a type $B$ spectrum, and germinated spores. The absorption bands marked with an arrow in $c$ and $d$ appear in the spectrum of the extracted lipid $b$.

specimens. This technique and its limitations have been discussed by Robinson (1952); Bellamy (1953); Martin (1957) and Bartlet (1957). The difference spectrum between vacuolated vegetative organisms and germinated spores is shown in Fig. $5 c$, the absorption bands marked with an arrow appear in the spectrum of the extracted lipid (Fig. $5 b$ ). A large part of the difference between vacuolated vegetative organisms and germinated spores can thus be attributed 
to the presence of the lipid in the vacuolated organisms. We used germinated spores as our reference material because they are almost free from calcium dipicolinate and lipid. Absorption bands attributable to the lipid do not appear in the spectra of our strains of Bacillus subtilis. This is in accord with Lemoigne's report that he was able to extract only $0.029 \%$ (dry weight) of his 'lipide' from this species.

We observed that the spectra obtained from a given sample of Bacillus megaterium when freshly prepared and after being kept for a time may not be identical. This effect occurs when dry films of bacteria on silver chloride are stored for more than $12 \mathrm{hr}$.; it occurs also in some heat-killed suspensions stored at $3^{\circ}$. The factors which determine whether or not a suspension changes on storage have not yet been found. Freshly harvested vacuolated organisms, whether alive or killed, have spectra which are like that in Fig. $6 a$ and for convenience this will be called a type $A$ spectrum. The spectrum of stored bacteria can change to that shown in Fig. $5 a$; this will be referred to as a type $B$ spectrum. The difference spectrum between organisms giving a type $\mathrm{B}$ spectrum and germinated spores (Fig. $5 d$ ) is similar to that of the extracted lipid. The bands marked with an arrow in Fig. $5 d$ appear in the spectrum of the extracted lipid.

Artificial mixtures of lipid and germinated spores have been made and from these it was shown that up to a concentration of $50 \%$ the ratio $\alpha$ is proportional to the amount of lipid in the preparation. The ratio $\alpha$ can thus be used to estimate the proportion of lipid in vegetative organisms. It has already been seen that $\alpha$ achieves a greater maximum for bacteria grown in shaken liquid medium than for bacteria from plate cultures. Thus growth in shaken liquid medium results in the production of a greater proportion of lipid. This is in accordance with our yields of extracted lipid and with the work of Lemoigne (1946).

When a washed suspension of vacuolated organisms is allowed to autolyse the suspension becomes acid and loses all the bands characteristic of the lipid (Fig. $6 b$ ). The acidity, according to Lemoigne, is due to depolymerization of the 'lipide' to $\beta$-hydroxybutyric acid. This is confirmed by the difference spectrum between autolysed vacuolated vegetative organisms and germinated spores (Fig. $6 c$ ) which contains nearly all the absorption bands in the spectrum of $\beta$-hydroxybutyric acid (Fig. $6 d$ ).

Powell (1953) observed that when resting spores of Bacillus megaterium germinated, there appeared in the medium a large amount of the calcium salt of pyridine-2:6-dicarboxylic acid (dipicolinic acid). We have obtained the spectra of the free acid, its sodium salt, its calcium salt (Fig. $7 d$ ) and its monoethyl and diethyl esters. The latter were examined because of the suggestion by Perry \& Foster (1956) that the dipicolinic acid is present in the spore as the monoethyl ester.

The spectra of resting and germinated spores of Bacillus megaterium are shown in Fig. $7 a$ and $b$. The difference spectrum between resting and germinated spores (Fig. 7c) is much more like the spectra of the calcium and sodium salts of dipicolinic acid than that of the free acid or of either of the esters. Since 


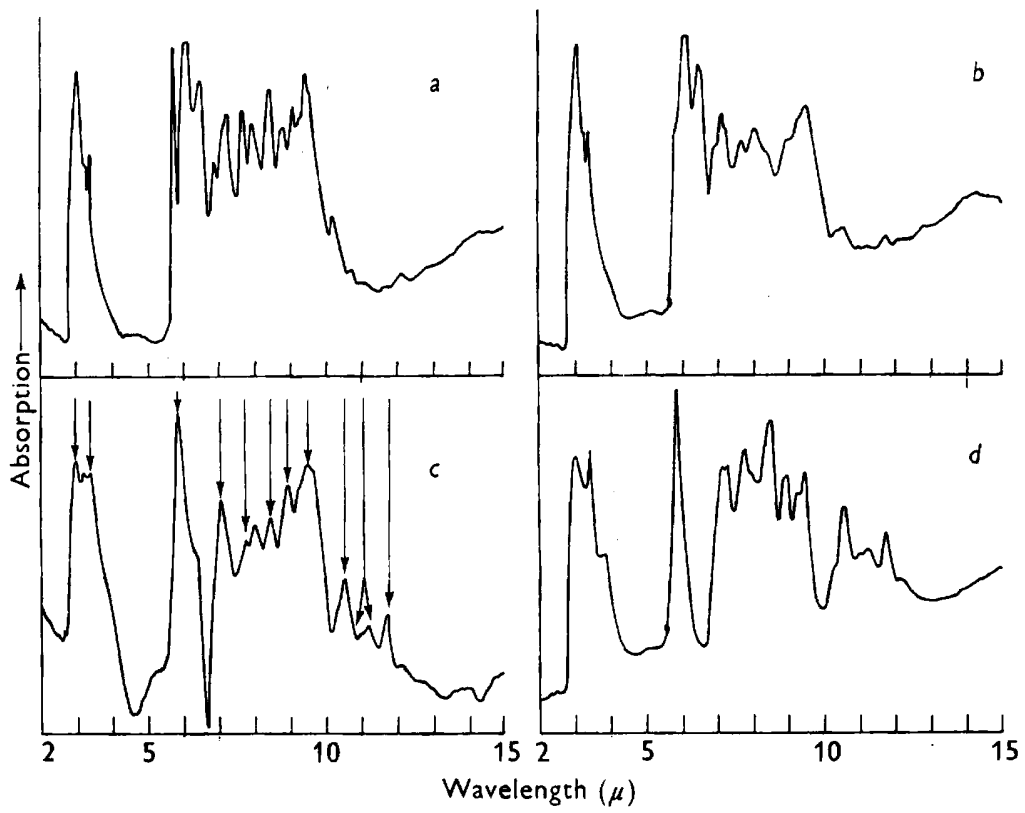

Fig. 6. Infrared absorption spectra : $a$, type A spectrum of vacuolated vegetative organisms (Bacillus megaterium); $b$, autolysed vacuolated vegetative organisms (B. megaterium); $c$, the difference spectrum between autolysed vacuolated vegetative organisms and germinated spores (B. megaterium); $d, \beta$-hydroxybutyric acid (film of pure liquid between sodium chloride plates). The absorption bands marked with an arrow in $c$ appear in the spectrum of $\beta$-hydroxybutyric acid $d$.

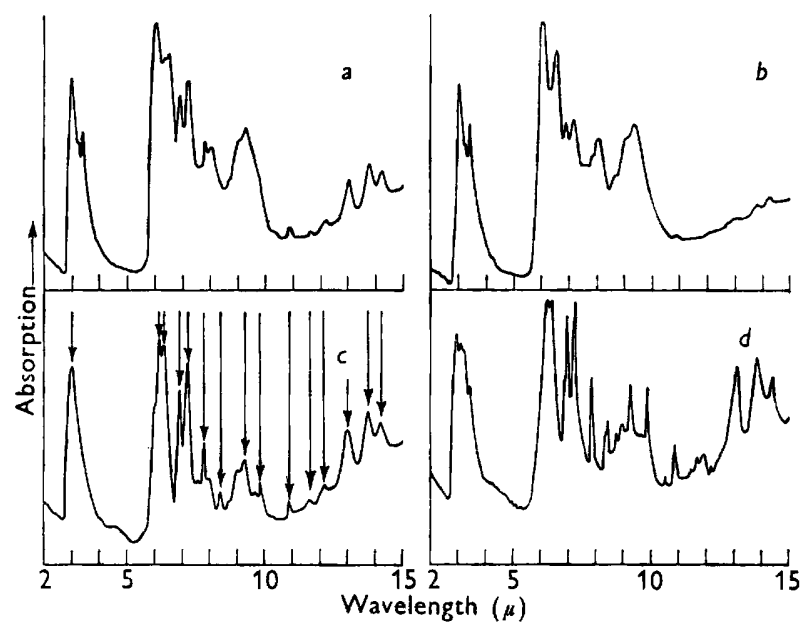

Fig. 7. Infrared absorption spectra: $a$, resting spores of Bacillus megaterium; $b$, germinated spores of $B$. megaterium; $c$, the difference spectrum between resting and germinated spores of $\boldsymbol{B}$. megaterium; $d$, calcium salt of dipicolinic acid (potassium chloride pressed disk). The absorption bands marked with an arrow in $c$ appear in the spectrum of the calcium salt of dipicolinic acid $d$. 
calcium dipicolinate appears in the medium it is likely that dipicolinic acid is present in the spore largely as the calcium salt. If the resting spore contains the ester then it is in very small amounts.

\section{DISCUSSION}

The infrared spectra of Bacillus megaterium undergo a cyclic change and are most complex when the organisms of the sample show maximum vacuolation when stained with the spore stain and maximum uptake of sudan black. The main effect of varying parameters such as the medium, temperature of incubation, inoculum size and resistance to drugs is to alter the time scale of the development of the culture.

The curves of Fig. 4, which show the variation of the ratio $\alpha$ with duration of incubation for different population densities, the way in which the change of $\alpha$ with time is affected by the addition of glucose to the medium and the relationship between the change of $\alpha$ and the change of dry weight can be explained by one hypothesis, namely: that the bacteria do not accumulate lipid when they are actively dividing; that only when division has stopped do they increase in both size and lipid content. To test this explanation it will be necessary to observe the changes taking place in individual organisms, by using a culture chamber on a microscope (Powell, 1956).

At the stage of morphological development which we have described as vacuolated vegetative, the organisms contain several dark granules when stained with sudan black, and large refractile inclusions under phase-contrast illumination. The ratio $\alpha$ obtained from the spectra of such organisms shows that they contain a large amount of lipid. We have failed to extract the lipid from intact bacteria by fat solvents and have demonstrated that there is little or none in the cell walls. It seems likely that the vacuoles are the same as the granules which stain with sudan black, are the refractile bodies, and contain the lipid within the bacterium. The identity of the vacuoles and the granules has been suggested by Burdon, Stokes \& Kimbrough (1942) but still awaits proof.

It appears that in the extraction process and in the ageing of the bacteria some change takes place in the lipid component. The difference between type $A$ and type $B$ spectra is very much smaller than the difference between the spectra of vacuolated and non-vacuolated organisms. The process underlying the change and its possible connexion with the degree of polymerization of the lipid must be the subject of further work. The methods we used to extract the lipid are rather severe but many authors, among them Lemoigne (1926) and Starkey (1946), recognized that acid hydrolysis or mechanical rupture of the cell wall was a necessary preliminary to the removal of fat from the bacterium.

Kull \& Grimm (1956) recorded the spectra of bacterial strains trained to be resistant to certain antibiotics and compared them with the spectra of the sensitive parent strains. They found that there were qualitative differences between the spectra of resistant and sensitive strains of Bacillus megaterium and Mycobacterium tuberculosis, whereas the spectra of the resistant variants 
of several other species showed no significant differences from those of the corresponding parent strains. In view of the large changes in spectra which result from altering the cultural conditions and since Kull \& Grimm did not describe their experimental technique in sufficient detail, it is difficult to assess the significance of their conclusions. Our experience suggests that their results can be explained by a change in the time scale of the development of the culture.

Blackwood \& Epp (1957) grew Bacillus megaterium on a nutrient medium containing glucose and obtained infrared spectra of the resulting cultures. Their spectra are similar to our type A spectra yet they conclude that $\beta$-hydroxybutyric acid accumulates in the bacteria. Our difference spectra make it clear however, that in freshly harvested bacteria it is not $\beta$-hydroxybutyric acid which is responsible for the complexity of the spectrum, but the lipid in substantially the same form as is obtained by extraction. When the suspension contains the free acid the spectrum has lost its complexity (Fig. 6b).

Efforts made to bring the aerobic spore-forming bacilli into a system of classification based upon their infrared absorption spectra have not as yet been successful, although Blackwood \& Epp (1957) hold out the hope that it may be possible. The large variations in the spectra of vegetative organisms which result from altering the conditions under which the bacteria are grown are much greater than any due to species or strain differences. Originally we thought that cultures grown on media which inhibit spore formation, such as tryptic meat (TM) agar, might be used for identification but as has already been seen cultures harvested from TM agar undergo the full change in spectrum.

In any sample of vegetative organisms of the aerobic spore-forming bacilli harvested from solid or liquid medium there is considerable diversity of morphological forms. As these different forms have different infrared absorption spectra the spectrum of the whole sample depends upon the proportions of the morphological forms in the specimen. Bacillus megaterium grown on glucose lactate agar is relatively uniform in appearance at any stage of the growth cycle, but other species grown under similar conditions are relatively heterogeneous. This heterogeneity is not easily controlled and the simple procedure that we used to obtain the spectra of strains representative of other genera is not adequate to give specimens which appear morphologically homogeneous. The method used by Halvorson (1957) may be necessary to obtain synchronous growth. The variability introduced into the spectra because of these effects is of the same order of magnitude as the differences between the spectra of species in other genera. The only reproducible stage in the growth cycle is the resting spore. We have found that the spectrum of washed resting spores is not influenced by the medium on which the spores are produced.

Most spore suspensions are contaminated to a variable extent with vegetative organisms, lysed material and cell debris. A small amount of such contamination has a noticeable effect: the spectra of washed spores and of spores slightly contaminated with vegetative organisms are apparently different. The spectra of pure clean resting spore suspensions may make differentiation between species possible. So far the practical difficulty of preparing pure clean suspensions has prevented us from deciding whether or not this may be so. 
In the past numerous attempts have been made to classify the Bacillus group on the basis of fat storage (Burdon et al. 1942; Lemoigne, Delaporte \& Croson, 1944; Imšenecki, 1945; Burdon, 1946). The validity of the method has recently been assessed by Smith, Gordon \& Clark (1952) who concluded that this criterion must be used with discretion and not as a definite basis on which to separate certain species from others. Their method was entirely qualitative, being based upon fuchsin-stained films. The infrared method can be made quantitative and therefore may eventually be of some use in differentiating between species. We are forced to the conclusion therefore that the infrared method cannot yet be used as a basis of differentiating between species of the aerobic spore-forming bacilli.

We thank Dr D. W. Henderson, Director, M.R.E. for his advice and criticism. We are indebted to Mrs Joan F. Powell for supplying us with samples of freezedried bacteria and of pyridine-2:6-dicarboxylic acid and its derivatives; and to the Distillers Company Ltd. for a gift of bacitracin. We are grateful also to Mr F. A. Dark for the differential centrifugation which gave us cell wall and lipid samples, to Mr H. E. Wade for phosphorus estimations, to Mr S. Bailey for the preparation of the monoethyl ester of dipicolinic acid and to Miss Jean M. Scott and Mr A. A. Dunthorn for their technical assistance. Finally, we wish to thank Mr E. O. Powell for his constructive criticism of the work and the manuscript.

\section{REFERENCES}

BARTLET, J. C. (1957). Identification of edible oils and the detection of oil adulteration by differential infrared spectroscopy. Nature, Lond. 180, 1071.

Bayne-Jones, S. \& Petrilli, A. (1933). Cytological changes during the formation of the endospore in Bacillus megaterium. J. Bact. 25, 261.

BeLlamy, L. J. (1953). Analysis with double-beam infra-red spectrometers. J. appl. Chem. 3, 421 .

BLACKWOOD, A. C. \& EPP, A. (1957). Identification of $\beta$-hydroxybutyric acid in bacterial cells by infrared spectrophotometry. J. Bact. 74, 266.

Burdon, K. L. (1946). Fatty material in bacteria and fungi revealed by staining dried, fixed slide preparations. J. Bact. 52, 665 .

Burdon, K. L., Stokes, J. C. \& Kimbrough, C. E. (1942). Studies of the common aerobic spore-forming bacilli. I. Staining for fat with sudan black B-safranin. J. Bact. 43, 717.

Fond, M. A. \& Wilkinson, G. R. (1954). The preparation and properties of pressed alkali halide disks with special reference to their use in spectroscopy. $J, s c i$. Instrum. 31, 338.

Greenstreet, J. E. S. \& Norris, K. P. (1957). The existence of differences between the infrared absorption spectra of bacteria. Spectrochim. Acta, 9, 177.

Halvorson, H. O. (1957). Rapid and simultaneous sporulation. J. appl. Bact. $20,305$.

Harman, T. L. (1940). The use of sudan black B as a bacterial fat stain. Stain Tech. 15, 23.

Haynes, W. C., Melvin, E. H., Locke, J. M., Glass, C. A. \& Senti, F. R. (1958). Certain factors affecting the infrared spectra of selected microorganisms. Appl. Microbiol. 6, 298.

HEwITT, L. F. (1951). Effect of cultural conditions on bacterial cytology. J. gen. Microbiol. 5, 293.

Hills, G. M., Belton, F. C. \& Blatchley, E. D. (1949). Ayfivin: Production in chemically defined media and comparison with licheniformin. Brit. J. exp. Path. 30, 427. 
IMŠENECKI, A. (1945). On the structure of anaerobic bacteria. J. Bact. 49, 1.

Kenner, B. A., Riddie, J. W., Rockwood, S. W. \& Bordner, R. H. (1958). Bacterial identification by infrared spectrophotometry. II. Effect of instrumental and environmental variables. J. Bact. 75, 16.

KNaYSI, G. (1946). On the existence, morphology, nature and function of the cytoplasmic membrane in the bacterial cell. J. Bact. 51, 113.

Kuld, F. C. \& Grimm, M. R. (1956). Differentiation of resistant mutants by infrared analysis. J. Bact. 71, 342.

Lemoigne, M. (1923). Production d'acide $\beta$-oxybutyrique par certaines bactéries du group du B. subtilis. C.R. Acad. Sci., Paris, 176, 1761.

Lemoigne, M. (1926). Produits de deshydration et de polymérisation de l'acide $\beta$-oxybutyrique. Bull. Soc. Chim. biol., Paris, 8, 770.

Lemoigne, M. (1946). Fermentation $\beta$-hydroxybutyrique (Formation d'acide $\beta$-hydroxybutyrique par autolyse). Helv. chim. acta, 29, 1303.

Lemoigne, M., Delaporte, B. \& Croson, M. (1944). Contribution a l'étude botanique et biochemique des bactéries du genre Bacillus. Valeur du test des lipides $\beta$-hydroxybutyriques pour la characterisation des espèces. Ann. Inst. Pasteur, 70, 224.

Levine, S., Stevenson, H. J. R., Bordner, R. H. \& Edwards, P. R. (1955). Typing of Klebsiella by infrared spectrophotometry. J. infect. Dis. 96, 193.

Lewis, I. M. (1934). Cell inclusions and endospore formation in Bacillus mycoides. J. Bact. $28,133$.

Martin, A. E. (1957). Difference and derivative spectra. Nature, Lond. 180, 231.

Mires, A. A. \& Mrsra, S. S. (1938). The estimation of the bactericidal power of the blood. J. Hyg., Camb. 38, 732.

Perry, J. J. \& Foster, J. W. (1956). Monoethyl ester of dipicolinic acid from bacterial spores. J. Bact. $72,295$.

Powell, E. O. (1956). An improved culture chamber for the study of living bacteria. J. R. micr. Soc. 75, 235.

Powell, J. F. (1950). Factors affecting the germination of thick suspensions of Bacillus subtilis spores in L-alanine solution. J. gen. Microbiol. 4, 330.

Poweld, J. F. (1953). Isolation of dipicolinic acid (Pyridine-2:6-dicarboxylic acid) from spores of Bacillus megaterium. Biochem. J. 54, 210.

Powell, J. F. \& Strange, R. E. (1956). Biochemical changes occurring during sporulation in Bacillus species. Biochem. $J .63,661$.

Randall, H. M., Smith, D. W., Colm, A. C. \& Nungester, W. J. (1951). Reproducibility of extracts of $M$. tuberculosis as determined by infrared spectroscopy. Amer. Rev. Tuberc. 63, 372.

Riddle, J. W., Kabler, P. W., Kenner, B. A., Bordner, R. H., Rockwood, S. W. \& Stevenson, H. J. R. (1956). Bacterial identification by infrared spectrophotometry. J. Bact. 72, 593.

RoBInson, D. Z. (1952). Quantitative analysis with infrared spectrophotometers. Differential analysis. Analyt. Chem. 24, 619.

Smith, N. R., Gordon, R. E. \& Clark, F. E. (1952). Aerobic sporeforming bacteria. U.S. Dept. of Agriculture. Agriculture Monograph No. 16.

STARKEY, R. L. (1946). Lipid production by a soil yeast. J. Bact. 51, 33.

Stevenson, H. J. R. \& BoldUan, O. E. A. (1952). Infrared spectrophotometry as a means for identification of bacteria. Science, 116, 111 .

Szybatski, W. \& Bryson, V. (1952). Genetic studies on microbial cross resistance to toxic agents. J. Bact. 64, 489 .

Thomas, L. C. \& Greenstreet, J. E. S. (1954). The identification of micro-organisms by infrared spectrophotometry. Spectrochim. Acta, 6, 302. 